\title{
In vitro Antimicrobial Evaluation of Ortho-/meta-Alkoxyphenylcarbamic Acid Esters Containing 4-(Pyrimidin-2-yl)piperazin-1-yl Moiety
}

\author{
Ivan Malik ${ }^{1}$, Eva Sedlarova ${ }^{1}$, Marian Bukovsky ${ }^{2}$, J ozef Csollei ${ }^{1,3}$, \\ Lukas Stanzel $^{1}$ and Lubica Sichrovska ${ }^{1}$
${ }^{1}$ Department of Pharmaceutical Chemistry, Faculty of Pharmacy, Comenius University, Odbojárov 10, 83232 Bratislava, Slovak Republic
${ }^{2}$ Department of Cell and Molecular Biology of Drugs, Faculty of Pharmacy, Comenius University, Kalinčiakova 8, Bratislava 832 32, Slovak Republic Palackého 1-3, 61242 Brno, Czech Republic \\ ${ }^{3}$ Department of Chemical Drugs, Faculty of Pharmacy, University of Veterinary and Pharmaceutical Sciences,
}

Received: March 06, 2014; Accepted: April 24, 2014; Published (web): July 06, 2014

The history of mankind can be regarded from a medical point of view as a struggle against infectious diseases. Infections were leading cause of death worldwide at the beginning of the $20^{\text {th }}$ century. ${ }^{1}$ Antimicrobial resistance due to over exposure to antimicrobials, is widely acknowledged as a major health threat. Alarmingly, resistance have increased significantly during the last decade. ${ }^{2-4}$ Moreover, another reason of the antimicrobial-drug resistance concern is its economic impact on physicians, patients, health-care administrators, pharmaceutical producers and the public. ${ }^{5,6}$

Antimicrobial-resistant pathogens affect patient outcomes in different ways. Resistant genes can alter the fitness of a bacterial pathogen, making it more or less virulent. The presence of resistance in a bacterial pathogen can lead to a delay in the administration of appropriate antimicrobial therapy and the antimicrobial therapies required to treat resistant pathogens can be toxic or inadequate. ${ }^{7}$ The overview ${ }^{1}$ has given insight in many therapeutic possibilities and approaches that have been used in the treatment of bacterial infections and in continuous battle

Correspondence to: Ivan Malik

Tel.: +421-2-50 117 226; Fax: +421-2-50 117 100;

E-mail: malikivan001@gmail.com

Dhaka Univ. J. Pharm. Sci. 13(2): 215-219, 2014 (December) between resistance development and overriding mechanisms. Similarly, numerous antifungal drugs with various chemical structures and scaffolds have appeared. ${ }^{8}$ However, their clinical use have been limited by the emergence of drug resistance, high risk toxicity, insufficiences in their antifungal activity and undesirable side effects. Therefore, to prevent the emergence and dissemination of resistant bacteria and fungi, continuing efforts to develop new antimicrobial agents are warranted. Searching for novel structures that the microorganisms have very rarely been presented with before, the aim of the present research was to in vitro evaluate an antimicrobial profile of newly prepared set of alkoxyphenylcarbamic acid esters containing 4(pyrimidin-2-yl)piperazin-1-yl fragment within their structure and try to identify some structural features which could be beneficial in terms of their antimicrobial potency.

The molecules under the study 1-4 (Table 1), chemically 1-[3-(2-/3-alkoxyphenylcarbamoyloxy)-2hydroxypropyl]-4-(pyrimidin-2-yl)piperazinium chlorides, were obtained from Department of Pharmaceutical Chemistry, Faculty of Pharmacy, Comenius University in Bratislava, Slovak Republic.

Their antimicrobial profile was investigated against Gram-positive bacteria S. aureus ATCC 6538 (Micrococcaceae), Gram-negative bacteria E. coli 
CNCTC 377/79 (Enterobacteriaceae) and yeast $C$. albicans CCM 8186 as well. The tested bacterial strains were purchased from American Type Culture Collection (Manassas, United States of America) and Czech National Collection of Type Cultures (Prague, Czech Republic); yeast was obtained from Czech Collection of Microorganisms (Brno, Czech Republic).
For a cultivation of the microorganisms, listed in the previous section of this short communication, a blood agar, Endo agar and Sabouraud's agar (Imuna, Slovak Republic) were used. Blood agar has been prepared by adding $10 \%$ of defibrine sheep's blood to melted and cooled $\left(50^{\circ} \mathrm{C}\right)$ competent components.

Table 1. The activity of the in vitro investigated compounds 1-4 against some microbial strains expressed by MIC values (mg/ml).

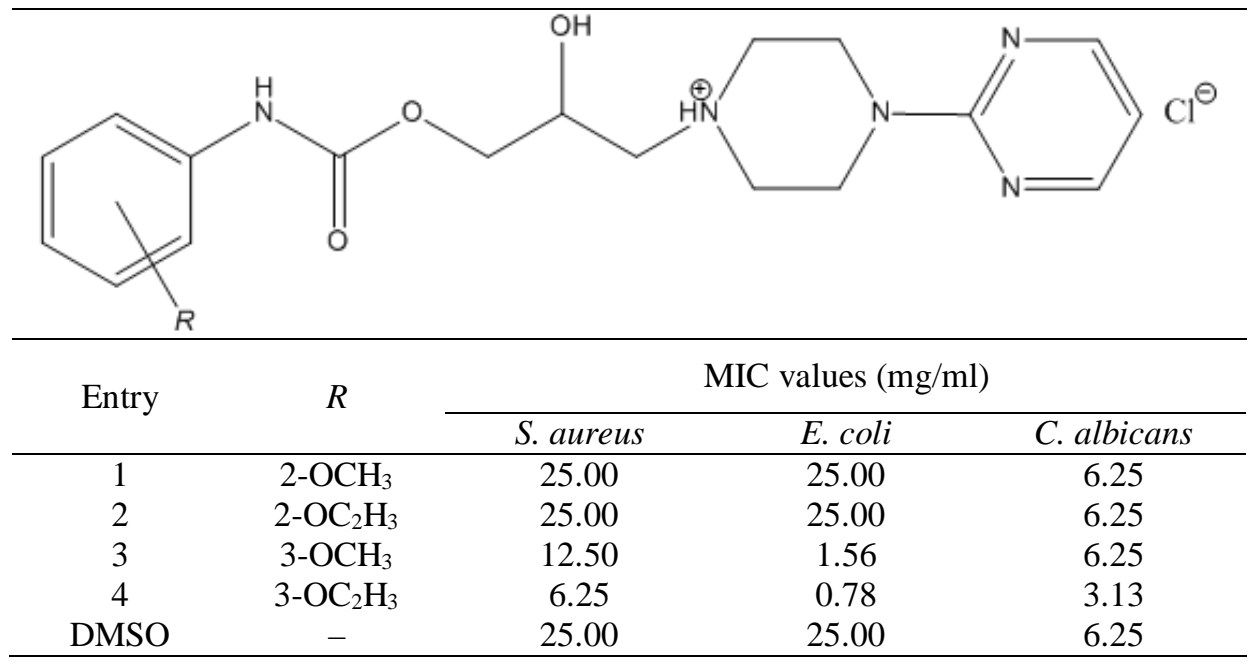

The minimum inhibitory concentration values (MIC) of inspected derivatives 1-4 were carried out by following the procedure previously published in paper. ${ }^{9}$ The respective tested molecules were dissolved in dimethylsulfoxide (DMSO; Merck, Germany) due to their very limited solubility in distilled water. Standard suspension of bacteria was prepared from their $24 \mathrm{~h}$ cultures which were cultivated on a blood agar (Gram-positive bacteria) and Endo agar (Gram-negative bacteria). Standard suspension of Candida was prepared from its $48 \mathrm{~h}$ cultures cultivated on Sabouraud's agar.

Prepared suspension contained the concentration of $5 \times 10^{7}$ colony forming unit (CFU) per $\mathrm{ml}$ of bacteria and $5 \times 10^{5} \mathrm{CFU} / \mathrm{mL}$ of Candida, respectively. The UV/VIS spectrophotometry was used for the determination of the microorganisms concentration, all evaluated suspensions were adjusted to the absorbance output of 0.35 at the wavelength of $540 \mathrm{~nm}$.
The suspension of microorganisms was added in the amount of $5 \mu \mathrm{l}$ into the solutions of inspected compounds $(100 \mu \mathrm{l})$ and to double concentrated peptone broth medium (8\%) for bacteria or to Sabouraud's medium (12\%) for Candida. The peptone broth and Sabouraud's media were purchased from Imuna (Slovak Republic).

Starting concentration of prepared stock solutions was $50.00 \mathrm{mg}$ of respective compound per $\mathrm{ml}$ of distilled water. These stock solutions (5\%) were then serially diluted by a half and final concentrations were $25.00,12.50,6.25,3.13,1.56$, 0.78 and $0.39 \mathrm{mg} / \mathrm{ml}$, respectively. Antibacterial effect of present DMSO in thus diluted final testing medium was completely lost.

The quantitative screening was performed using sterile 96-well plastic microtiter plates (with roundbottomed wells) with matching covers. Microorganisms were incubated in each well at $37{ }^{\circ} \mathrm{C}$ 
for $24 \mathrm{~h}$. Upon completion of this process, the volume of $5 \mu \mathrm{L}$ of evaluated suspension has been taken from each well by using transferring tool and cultured on a blood agar (S. aureus), Endo agar ( $E$. coli) or on Sabouraud's agar (C. albicans), respectively. Petri dishes were then incubated for 24 h at $37^{\circ} \mathrm{C}$.

Positive control using only an inoculation of the microorganisms and negative control using only DMSO were realized in parallel. Both DMSO and nutrient concentrations remained stable in each well, only the concentration of inhibitory compound has changed. All experiments were performed in duplicate. The MIC was regarded as the lowest concentration of antimicrobial agent required to inhibit the visible growth of microorganism after an incubation. ${ }^{10}$ The MIC was dependent on the presence/absence of the culture on used solid media after the transfer of $5 \mu \mathrm{L}$ of suspension from each well. The values of MIC which were estimated for tested compounds as well as for DMSO (due to comparison) are reported in Table $1 \mathrm{in} \mathrm{mg} / \mathrm{mL}$ units.

Following chemical structure of currently in vitro investigated compounds 1-4 (Table 1), their potency against $S$. aureus, E. coli and C. albicans might be influenced, among others, by: (i) orthoImeta-alkoxy side chain isomerism, (ii) their lipohydrophilic profile, (ii) nature of the fragment forming basic compartment. Possible impacts of aforementioned factors will be discussed in the parts below.

As current results clearly indicated (Table 1), meta-alkoxy substituted derivatives (the substituent $R$ attached to the position 3) were more active against $S$. aureus and $E$. coli than the ortho-alkoxy substituted ones (the substituent $R$ in the position 2 ). The explanation of such phenomenon could be that the proximity of attached ortho-alkoxy string to carbamate bond led to the twist of the phenyl ring plain towards the carbamate group. Described process resulted in the planarity violation of such molecule to provide subsequent conjugation of $\pi$ bonds of aromatic ring over $\mathrm{NH}$ fragment up to carbonyl group. The outcome of outlined conjugation was the different electronic density (charge) at carbonyl moiety which could be one of the possible binding sites to the reactive membrane locations. Considering meta-alkoxy substituted compounds, given secondary steric effect did not manifest.

The increase in the lipophilicity within the tested set of the substances 1-4, i.e. the elongation of attached alkoxyl, did not lead to more potent orthoalkoxy substituted molecules against all selected microbial strains. On the other hand, slight improvement in the efficiency was observed when investigating the potency of the derivatives bearing meta-alkoxy side chain.

The influence of the basic fragment selection on the activity in vitro will be discussed within next paragraphs. The presence of 4-(pyrimidin-2yl)piperazin-1-yl heterocycle in the structure would be relatively promising in terms of the efficiency against E. coli. On the other hand, the incorporation of such moiety was considered inappropriate for the improvement of the potency against $S$. aureus and $C$. albicans as well, as the data in Table 1 unambiguously documented.

The efficiency against $S$. aureus. Following previously estimated MICs related to structurally similar molecules, ${ }^{11}$ the introduction of 4-(2methylphenyl)piperazin-1-yl group instead of 4-(pyrimidin-2-yl)piperazin-1-yl led to lower MIC readouts of corresponding meta-alkoxy positional isomers. It could be suggested that the differences in assumed electronic and hydrophobic interactions involved by the substituents attached to piperazin1,4-diyl would be decisive for an antimicrobial profile of such alkoxyphenylcarbamic acid-based compounds. In the structure of currently tested derivatives, both atoms of nitrogen have shown the ability to polarize the $\pi$-electron system of pyrimidin2 -yl, resulting in slightly decreased electron density on the ring carbons. It seemed that the presence of relatively $\pi$-electron rich aromatic was vital for the activity maintenance against given Gram-positive bacterial strain. Supporting this argument, previously investigated structures which basic part was formed by 4-(2-methylphenyl)piperazin-1-yl ${ }^{12}$ were 
considered more promising than the molecules 1-4 because of the presence of methyl moiety which released electron density to aromatic system by the stabilizing interaction between a $\mathrm{C}-\mathrm{H}$ bond (of methyl group) and an adjacent of $\sigma$-bond. ${ }^{13}$

The efficiency against $\boldsymbol{E}$. coli. Previously in vitro tested meta-alkoxyphenylcarbamic acid esters containing 4-(3-trifluoromethylphenyl)piperazin-1-yl fragment ${ }^{14}$ were completely inactive against given Gram-positive bacterial strain with its $\mathrm{MIC}=6.25$ $\mathrm{mg} / \mathrm{mL}$ regardless the number of carbon atoms which formed attached alkoxyl. This lipophilic substituent is also considered relatively sterically bulky and with relatively strong electron-withdrawing effect towards the aromate when attached. Given statement could be proved by the values of Hansch-Leo $\pi$ constant which was set to 0.88 for 3-trifluoromethyl (the readout of 0.00 was set to hydrogen atom). The electronwithdrawing potential of concerned moiety, expressed by the value of Hammett $\sigma_{\mathrm{m}}$ constant which was 0.43 (atom of hydrogen has shown the output of 0.00 ), is also evidently clear. ${ }^{15}$ Similarly, steric properties of this substituent were defined, among others, by the $B_{\mathrm{i}}-B_{\mathrm{iv}}$ and $L$ descriptors, respectively. More detailed information characterizing the parameters mentioned above can be found in the monography. ${ }^{15}$

Current research revealed that the replacement of such (substituted) phenyl ring by heterocyclic structure meant the improvement in the activity, as observed MIC data of 1.56 and $0.78 \mathrm{mg} / \mathrm{mL}$ indicated (Table 1). From chemical point of view, pyrimidine, as heterocyclic analogue of benzene, meets the Hückel criteria for an aromaticity. Such sixmembered unsaturated ring is planar, both nitrogens are $s p^{2}$-hybridized, it has one $2 \mathrm{p}$ orbital on each atom of the ring and six electrons in the $\pi$-system as well. An important parameter to keep track of in aromatic heterocycles is whether lone pairs of electrons are part of the aromatic $\pi$-system or not. In pyrimidine, none unshared pair of nitrogen electrons is part of the $\pi$-system, but each nitrogen contributes one electron to the aromatic $\pi$-system. ${ }^{16,17}$ Following mentioned, it could be suggested that the presence of aromatic moiety would be favorable in terms of the activity against E. coli. As published previously ${ }^{12}$, it can be presumed that electronic and hydrophobic interactions were primarily responsible for a better internalisation of the compounds under the study through E. coli outer membrane. Moreover, consequent electronic interactions with various membrane components of $E$. coli ( $\pi-\pi$ hydrophobic interactions) could be also taken into the consideration.

Compared to previously in vitro inspected compounds bearing 4-(3-trifluoromethylphenyl) piperazin-1-yl, the presence of given fragment caused the decrease in the electron density towards present aromatic system and, consequently, the loss of the activity.

The efficiency against $C$. albicans. Entire set of the compounds 1-4 was regarded as completely inactive against $C$. albicans with the MICs $>1.00$ $\mathrm{mg} / \mathrm{mL}$ (Table 1). Following current results and the conclusions from previously published research article $^{14}$, except of the lipophilicity enhancement and convenient steric properties, the presence of sterically bulkier substituent which has shown relatively strong electron-withdrawing effect within basic part of such alkoxyphenylcarbamic acid derivatives was considered the most important factor.

\section{ACKNOWLEDGEMENTS}

The authors are very grateful to Slovak Grant Agency for Science for supporting by the VEGA Grant Project No. 1/0039/12. The authors thank the anonymous reviewers for their valuable comments and helpful suggestions for revision.

\section{REFERENCES}

1. Bockstael, K. and van Aerschot, A. 2009. Antimicrobial resistance in bacteria. Cent. Eur. J. Med. 4, 141-155.

2. Giske, Ch.G. and Cornaglia, G., on behalf of the ESCMID Study Group of Antimicrobial Resistance Surveillance (ESGARS). 2010. Supranational surveillance of antimicrobial resistance: The legacy of the last decade and proposals for the future. Drug Resist. Updat. 13, 93-98.

3. European Antimicrobial Resistance Surveillance System. 2000. EARSS Annual Report. RIVM, Bilthoven, Netherlands. 
4. Byarugaba, D.K. 2004. A view on antimicrobial resistance in developing countries and responsible risk factors. Int. J. Antimicrob. Agents 24, 105-110.

5. Paladino, J.A., Sunderlin, J.L., Price, C.S. and Schentag, J.J. 2002. Economic consequences of antimicrobial resistance. Surg. Infect. 3, 259-267.

6. McGowan, Jr., J.E. 2001. Economic impact of antimicrobial resistance. Emerg. Infect. Dis. 7, 286-292.

7. Cosgrove, S.E. and Carmeli, Y. 2003.The impact of antimicrobial resistance on health and economic outcomes. Clin. Infect. Dis. 36, 1433-1437.

8. Kleemann, A., Engel, J., Kutscher, B. and Reichert, D. 2009. Pharmaceutical Substances: Syntheses, Patents, Applications of the Most Relevant APIs. $5^{\text {th }}$ Rev. Ed., Georg Thieme Verlag, Stuttgart, $1800 \mathrm{pp}$.

9. Malík, I., Bukovský, M., Andriamainty, F. and Gališinová, J. 2013. Antimicrobial effect of para-alkoxyphenylcarbamic acid esters containing substituted $N$-phenylpiperazine moiety. Br. J. Microbiol. 44, 457-463.

10. Andrews, J.M. 2001. Determination of minimum inhibitory concentrations. J. Antimicrob. Chemother. 48, 5-16.

11. Malík, I., Sedlárová, E., Bukovský, M., Csöllei, J. and Sichrovská, L. 2013. Basic esters of meta-/paraalkoxyphenylcarbamic acid containing 4-(2-methyl-/2fluorophenyl)piperazin-1-yl moiety and their antimicrobial activity. Fresen. Environ. Bull. 22, 2689-2694.
12. Sedlárová, E., Malík, I., Bukovský, M., Csöllei, J. and Sichrovská, L'. 2013. Basic ortho-alkoxyphenylcarbamic acid esters containing variously substituted $\mathrm{N}$-phenylpiperazine fragment and their antimicrobial activity. Fresen. Environ. Bull. 22, 1603-1608.

13. Hepworth, J.D., Waring, D.R., Waring, M.J., Berry, M. and Drayton, C. 2002. Aromatic Chemistry, The Royal Society of Chemistry, Cambridge, 176 pp.

14. Malík, I., Bukovský, M., Andriamainty, F. and Gališinová, J. 2012. Antimicrobial activity of meta-alkoxyphenylcarbamates containing substituted $N$-phenylpiperazine fragment. Br. J. Microbiol. 43, 959-965.

15. Kubinyi, H. 1993. QSAR: Hansch Analysis and Related Approaches, Wiley-VCh Verlag, Weinheim, $240 \mathrm{pp}$

16. Brown, W.H., Foote, Ch.S., Iverson, B.L. and Anslyn, E.V. 2012. Organic Chemistry, $\sigma^{\text {th }} E d$., Brooks/Cole Cengage Learning, Belmont, 1303 pp.

17. McMurry, J. 2011. Organic Chemistry, $8^{\text {th }}$ Ed., Thomson Learning, London, 1376 pp. 